\title{
The impact of driving homogeneity due to automation and cooperation of vehicles on uphill freeway sections
}

\author{
Michail Makridis $^{1 *}$, Ludovic Leclercq² ${ }^{2}$ Konstantinos Mattas ${ }^{1}$ and Biagio Ciuffo ${ }^{1}$
}

\begin{abstract}
Background: This work presents a microsimulation study on the topic on an uphill network, regarding the potential impact of AVs and Cooperative-AVs (Coop-AVs or CAVs), vehicles able to cooperate with the infrastructure. The novelty of the proposed approach is that the simulation of all vehicles is performed with a common hybrid car-following model that takes explicitly into account the variability in the vehicle dynamics and the driving behaviors.

Methods: Simulation of longitudinal movement of the individual vehicles is performed with a common hybrid carfollowing model that takes explicitly into account the variability in the vehicle dynamics and the driving behaviors. Different homogeneity levels in the vehicles and drivers are tested, while the cooperation is explicitly assessed by proposing a realistic Vehicle to Infrastructure (V2I) logic. Possible reduction in the response times of the vehicles is also studied.
\end{abstract}

Results: Results with more homogenous vehicle movements have more consistent performance in terms of traffic flow, that is independent of the order that the vehicles enter the network. Finally, the cooperation with the infrastructure can limit high variations in the vehicles' accelerations and thus potential traffic jams.

Conclusions: Homogenized flows can mitigate or even solve traffic-related problems related to the variability in driving behaviors, such as bottlenecks and stop-and-go waves.

Keywords: Vehicle to Infrastructure, Automated Vehicles, Vehicle Dynamics, Traffic simulation, Driving behavior, Traffic Flow

\section{Introduction}

Vehicle automation is progressively introduced in modern traffic networks with technologies that promise to transform the transport sector. New driver assistance technologies are constantly introduced and they will evolve up to a point in which, the complete dynamic driving task can be safely taken over by the vehicle, in every driving situation possible (i.e. the ultimate SAE level 5 full automation). However, achieving a sustainable transport system becomes more and more challenging given the expected increases in passenger and freight transport (a growth by about $42 \%$ and $60 \%$ from 2010 to 2050, respectively), road transport being the main transport mode used in the EU [6]. Automated Vehicle (AV) technology alone, will not necessarily be smarter than conventional vehicles driven by humans [1]. Introduction

\footnotetext{
* Correspondence: michail.makridis@ec.europa.eu;

michail.makridis@ivt.baug.ethz.ch

${ }^{1}$ European Commission, Joint Research Centre, Ispra, VA, Italy

Full list of author information is available at the end of the article
} 
of automated functionalities is not expected to break the existing interaction between traffic information and traffic conditions and heterogeneity due to the different vehicles' behavior will probably continue to exist, at least to a certain extent, as each vehicle manufacturer will bring to the market a different product [25]. An open question in the literature is how any anticipated reduction in the variability of the driving patterns can impact traffic flow.

Another essential dimension is the reaction time of the drivers or automated vehicles. Adaptive Cruise Control (ACC) system is already available in the market and currently, it is considered the closest proxy of full automation regarding the longitudinal movement [11]. In theory, automated functionalities have the advantage to instantly respond whenever needed, minimizing this way the reaction time, which for humans is more than $1 \mathrm{~s}[10,14]$. However, empirical studies on the estimation of the observable response time under non-critical conditions (referred here as response time) of Adaptive Cruise Control (ACC) systems currently available in the market proposed by $[19,20]$, show that the response time is lower but close to the corresponding human reaction times. It should be clarified that observable response or reaction time is different than brake reaction time [28]. This work considers reaction/response time as the time from the moment that a leader performs an action in the longitudinal direction (either acceleration or deceleration), until the moment that the follower reacts to it (either decelerating or accelerating) under the following conditions:

- The two vehicles are initially under stable car-following conditions with similar speeds.

- The ACC system in the following vehicle is on.

The observable response time can be higher than the vehicle's response time under unsafe or critical conditions, e.g. Emergency Braking. However, it directly impacts the traffic flow and can play a significant role where there are oscillations under high density flows leading to traffic instabilities.

A third dimension is related with the vehicles cooperation with the infrastructure. Vehicle automation offers new ways of automated standardized cooperation with the infrastructure that are not feasible with human driven vehicles [5].

Microsimulation modeling can offer valuable insights on the above dimensions but modeling the heterogeneity in driving behaviors is still a novel and open topic in the literature. Recently, the development of more precise models taking into account the vehicle dynamics and the driving aggressiveness offers new ways of assessment that are not based only on injection of stochasticity in car-following $[7,15,18]$.

In parallel, one of the common reasons for capacity bottlenecks in freeway networks is the sections uphill
[26]. Going uphill, drivers reduce speed while they enter the section. When the demand is high they don't compensate for the speed loss and most importantly the perturbation of the platoon leader creates a flow disturbance that it is propagated upstream, leading in cases to a traffic jam. Optimization of traffic flow at freeway uphill sections by controlling the vehicle acceleration recently attracts a lot of interest [8, 9]. Furthermore, in microsimulation studies regarding the impact assessment of connected and automated vehicles, the models used for simulation do not account for the reproduction of realistic vehicle dynamics $[22,23,29]$. In the literature, it has been mentioned that the absence of models capable to reproduce realistic vehicle power dynamics and driving behaviors in microsimulation, can lead to questionable results regarding both emissions-related studies and the study of traffic flow phenomena [2, 13, 15, 21].

For the above reasons, an uphill simulation environment using a vehicle-dynamics-based model and simulation of different driving styles accounting for different drivers is proposed and studied in this work. AVs and Coop-AVS are expected to reduce variability in driving patterns and vehicle dynamics and reduce the human observable reaction time. The main goal is to assess the potential impact of such technologies on the traffic flow of the future traffic networks and to showcase the potential benefits that will come from the cooperation of vehicles with the infrastructure (V2I). We use a simple one-lane segment and a constant grade uphill in a segment part. We propose a vehicle dynamics-based car-following model, which accounts for different driver behaviors and can be calibrated for different vehicle characteristics. Afterwards we perform simulations using the Lead Vehicle Problem principle $[3,4]$. The demand of the network consists of different drivers/vehicles from a pool of 125 profiles including both timid and aggressive driving styles. The simulations are repeated for different random seeds in order to validate the results. Since automation in the vehicle is expected to reduce variability in the driver domain, we perform the same simulations using a smaller pool of profiles for AVs and Coop-AVs, considering that each vehicle has its own driving style, similarly to existing commercial ACC systems for the longitudinal movement of the vehicle (assuming that each vehicle manufacturer implements its own ACC logic). Moreover, we propose a simple yet efficient control logic to showcase the impact of vehicle cooperation with the infrastructure (V2I). The control logic is motivated by the results presented by Goñi-Ros [8], identifying an acceleration maneuver uphill as the best strategy to minimize total delay and facilitate flow. Finally, the sensitivity of the results was tested using two different response times for $\mathrm{AVs}$ and CAVs, one equal to the reaction time of the human drivers and a second, reduced by $10 \%$ as it is empirically found in our previous work [18]. 
Results show that in the highly probable case that different manufacturers will deploy different implementations for their automated systems, the driving behavior variability introduced by the driver-vehicle duo in future networks will remain high. A reduction in the traffic delays can be expected, especially if the future ACC systems have response times lower than those of humans but the improvement will not be dramatic. On the other hand, cooperation of the vehicles with the infrastructure can play a significant role in future networks. Cooperation even on the basis of timid driving behavior can bring significant improvement in the network increasing the average speed and maximizing the flow. Finally, reduction of the reaction time implies faster reaction of the driver upon a perturbation event but also faster propagation of the perturbation information upstream and in this work reveals mixed results for different random seeds.

In the rest of the paper, the experimental setup is presented discussing the proposed car-following model for the simulation of the vehicles dynamics and driving behavior, the network and the generation of different driving profiles and the cooperation logic. Then, follows the description of proposed methodology, the results and finally the conclusions and future work.

\section{Experimental setup}

This section describes the experimental setup that was used as a benchmark for this simulation study. The rest of the section presents the model for the simulation of power dynamics and driving behaviors, the network, the algorithm for generation of variability in vehicle dynamics and driving style, the simulation of different vehicle types and the proposed cooperation control logic.

\subsection{The car-following model}

The proposed car-following model is based on the simplified Lagrangian Godunov scheme, which is described in the work of Leclercq [16] as follows:

$$
X(n, t+\Delta t)=\min \left(X(n, t)+v_{m} \Delta t,(1-\alpha) X(n, t)+\alpha X(n-1, t)-w \Delta t\right)
$$

where $v_{m}$ is the desired speed, $w$ is the wave speed, $k_{m}$ is the jam density and $a=w k_{m} \Delta t$. Equation 1 is the exact solution of the LWR model [17-19].

In this work, the lightweight Microsimulation Freeflow aCceleration model (MFC) [18] is incorporated within the framework of the LWR model bounding the free-flow acceleration based on the MFC output. More specifically, based on the definition given, the proposed model is:

$$
\begin{gathered}
q 1=X(n, t)+\Delta t * \min \left(v_{m}, V(n, t)+a_{M F C}\left(D S, G S_{t h}\right) \Delta t\right) \\
q 2=X(n-1, t)-w \Delta t \\
X(n, t+\Delta t)=\min (\mathrm{q} 1, \mathrm{q} 2)
\end{gathered}
$$

where $v_{m}$ is the desired speed, $a_{M F C}$ is the acceleration of the MFC model, $w$ is the wave speed, $k_{m}$ is the jam density here set to $0.15 \mathrm{veh} / \mathrm{m}$ and $a=1 . V(n, t)$ is speed of the vehicle $\mathrm{n}$ at time $t$.

The MFC takes as input common specifications of the vehicle, such as mass, gear ratio, maximum torque etc., which can be found available online and two parameters $\left(D S, G S_{t h}\right)$ taking values in the range $(0,1]$ in order to simulate different drivers. Parameter values closer to zero, indicate a timid driver, while when they take values closer to one, an aggressive one. More details can be found in [18].

Figure 1 illustrates the acceleration potential for the same vehicle and three different drivers as they are defined by the different values of $D S$ and $G S_{t h}$ parameters. The figure derives from the simulation of a free-flow acceleration from 0 $\mathrm{km} / \mathrm{h}$ to maximum speed for a commercial vehicle with a 9-speed automatic gearbox. The simulation is performed for three different drivers described by the different $(D S$, $\left.G S_{t h}\right)$ parameter sets. The figure on the top illustrates the acceleration over speed diagrams per driver from zero to the vehicle's maximum speed. The behavior of the MFC model can be perceived by the reader intuitively if we consider that the $D S$ parameter adjusts how high the acceleration on the Y-axis will be, while the will the $G S_{t h}$ parameter dictates how fast the driver will change gears jumping from a gear to another with higher (upshift) or lower (downshift) acceleration capability (X-axis). The figure on the bottom shows the speed over time trajectory during a free-flow acceleration from zero to a desired speed. The differences of the three drivers can be spotted both regarding the time they need to reach the same speed, and the speed oscillations due to the different gear shifting strategies. From the implementation point of view, the acceleration over speed curves shown in the figure can be precomputed, leaving the proposed model computationally inexpensive.

\subsection{Network description}

We consider a one-lane road segment with length of 1000 $\mathrm{m}$. Starting on $500 \mathrm{~m}$. we introduce an upgrade for $300 \mathrm{~m}$. The cooperation control logic is applied along a segment with length $500 \mathrm{~m}$ from $400 \mathrm{~m}$ to $900 \mathrm{~m}$, as shown in Fig. 2. The wave speed is set to $20 \mathrm{~km} / \mathrm{h}$, the jam density to $150 \mathrm{veh} / \mathrm{km}$. The effect of the upgrade on the vehicle acceleration is dictated by the following equation:

$$
a c c_{g}=\frac{F_{g}}{m}=\frac{-\left(9.81 * \sin (g) * m+F_{0} *(1-\cos (g))\right.}{m}
$$

where $g$ is the gradient of the road, $m$ is the vehicle's mass 

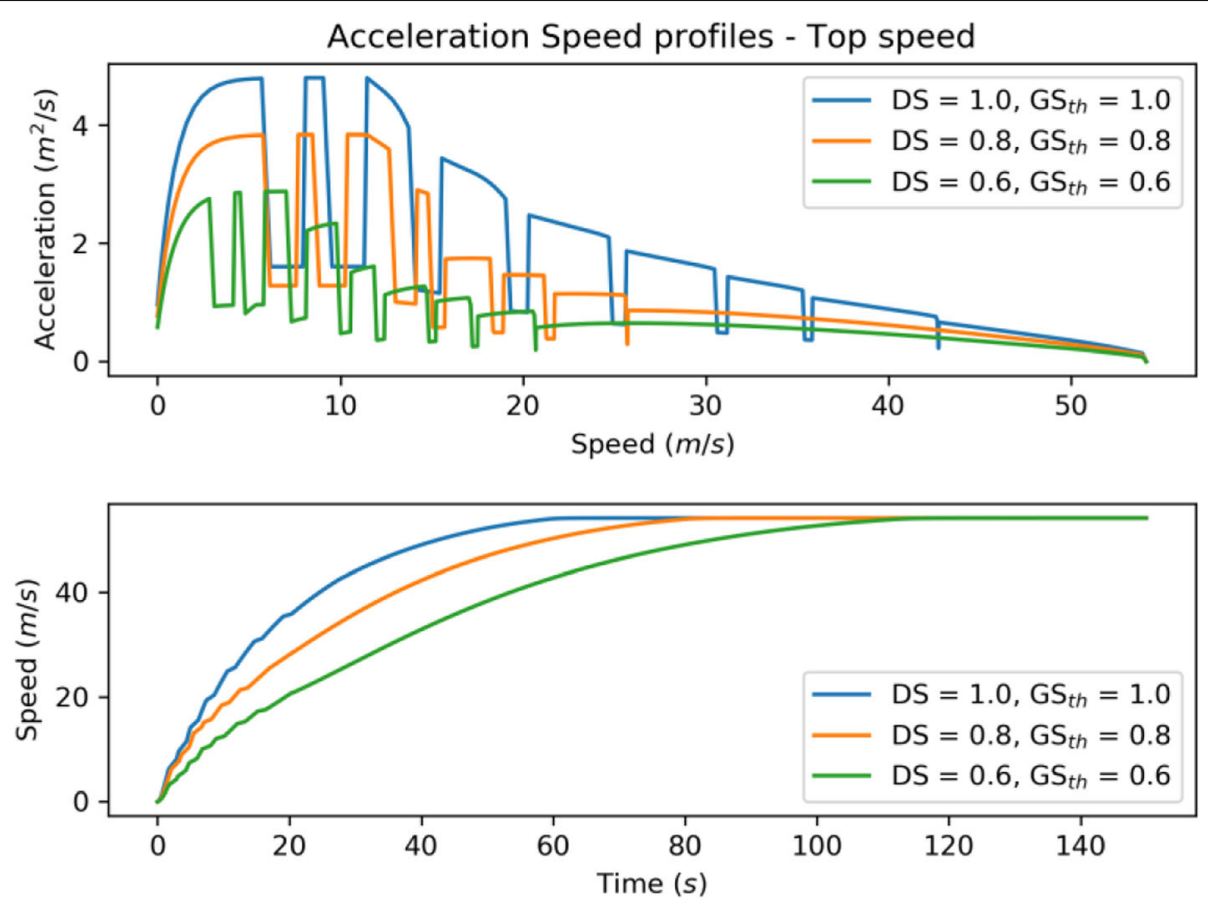

Fig. 1 Simulation of free-flow acceleration from $0 \mathrm{~km} / \mathrm{h}$ to maximum speed for a commercial vehicle with a 9-speed automatic gearbox. Top: Acceleration-Speed profiles for three different drivers with different driving styles. Bottom: The simulated speed profiles for the same drivers

and $F_{0}$ factor is commonly used to characterize the road load of vehicles and expresses the constant part of a vehicle's resistances (tire rolling resistances). A dedicated module for the calculation of the vehicle's road loads was developed by Tsiakmakis et al. [31] and was adopted for the needs of this study for the computation of $F_{0}$ and the free-flow acceleration output of the MFC model [18].

\subsection{Driving profile generation}

A driving profile is constructed and randomly assigned to each vehicle entering the network. Each individual driving profile is developed using the car technical characteristics and the two parameters $\left(D S, G S_{t h}\right)$ of the car-following model taking values in the range $(0,1]$ in order to simulate different drivers. Figure 3 illustrates the generation of different driving profiles (vehicle and driving parameters). The top row corresponds to five vehicle with different power specifications, the second row correspond to five $G S_{\text {th }}$ values that regulate how fast the driver changes gears (Xaxis) and the third row correspond to five $D S$ values that regulate the maximum desired acceleration (Y-axis). This work uses five different vehicles from car segments B and C (representative small and medium cars) [30] to cover the most common passenger cars segments in use in Europe in terms of mass, power, engine technology, and transmission. The driver characteristics derive from randomly selected values for the parameters $\left(D S, G S_{t h}\right)$. In general drivers with $\left(D S, G S_{t h}\right)$ values closer to 0 have more timid driving

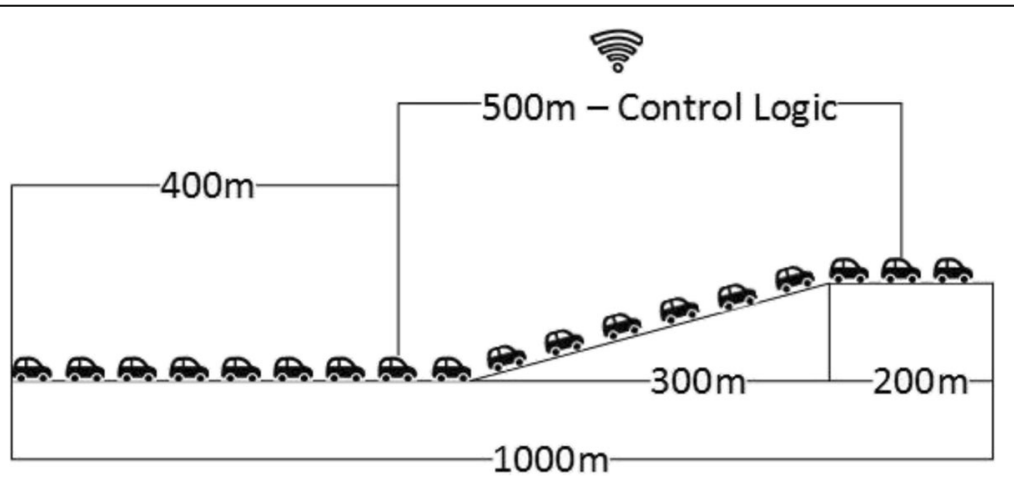

Fig. 2 One-lane network, $1 \mathrm{~km}$ long with an uphill segment of $300 \mathrm{~m}$. In the figure, it is shown also the area where the control logic is applied 


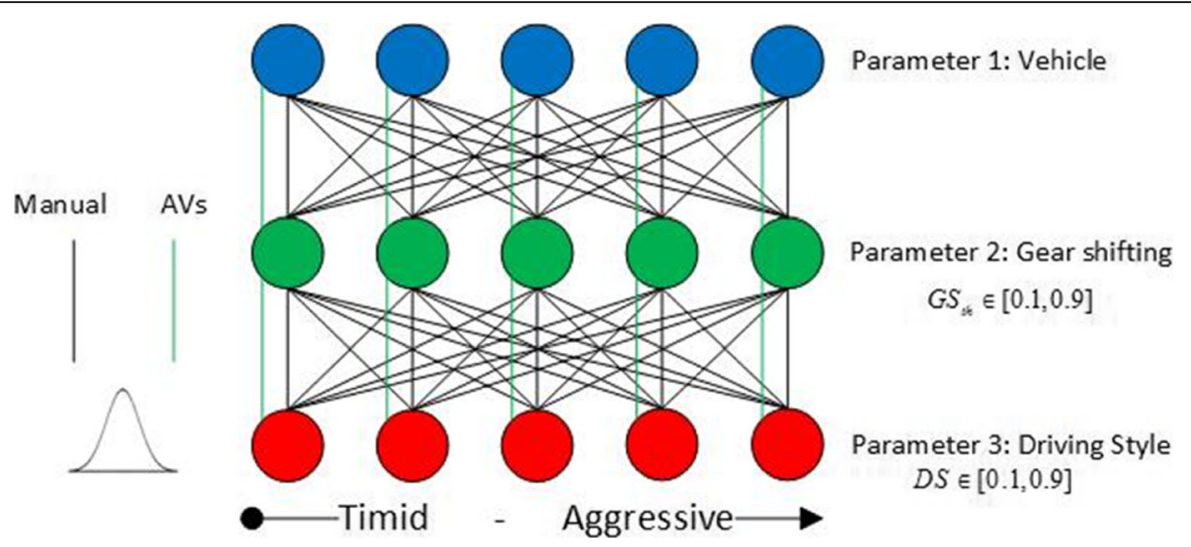

Fig. 3 Creation of 125 individual driving profiles $D P_{i}$ by choosing from a pool of 5 different cars, 5 gear shifting strategies and 5 driving styles. AVs are shown with green line and correspond to 5 individual profiles, one per each vehicle. The selection of the profiles during each simulation is performed based on a normal distribution around the average profile, i.e. the second value of each parameter

behavior, while drivers with $\left(D S, G S_{t h}\right)$ values closer to one, are more aggressive.

Consequently, each driving profile can be defined as follows:

$$
D P_{i}=\left\{C_{k}, G S_{t h, l}, D S_{m}\right\}
$$

where the $i$ driving profile refers to car $k$, with gear shifting value $l$ and driving style value $m$.

\subsection{Vehicle types}

All the simulation experiments involve three different types of vehicles, manually-driven (manual), automated vehicles (AVs) and cooperative automated vehicles (Coop-AVs). All types are simulated using the same carfollowing model described by Eq. 2. The difference lies in the variation of different driving styles that enter the network and the existence of cooperation or not with the infrastructure. More specifically:

\subsubsection{Human-driven vehicles (CVs)}

In simulation tests with $\mathrm{CVs}$, a random driving profile is assigned to each vehicle entering the network among all the possible combinations of $\left\{C_{k}, G S_{t h, l}, D S_{m}\right\}$ values as shown in Fig. 3 (125 in total).

\subsubsection{AVs}

In simulation tests with AVs, a fixed pair of assigned (DS, $G S_{t h}$ ) values is assigned to each of the five vehicles shown in the first row of Fig. 3. Consequently, each AV will have one of the five different driving profiles (green vertical lines).

\subsubsection{Coop-AVs}

Cooperative automated vehicles have exactly the same driving profiles as AVs. The difference lies that they behave according to the proposed cooperation control logic (see Section 2.5) for the network segment 400-900 $\mathrm{m}$ as illustrated in Fig. 2.

\subsection{Cooperation control logic}

In the literature, there are references that drivers underestimate high driving speeds under freeway conditions [32]. Variable Speed Limit (VSL) strategies have been used as a freeway metering mechanism or a homogenization scheme to reduce speed differences, which most probably derive from the variability in the vehicle specs and the driving style [27]. Finally, in uphill areas it has been observed that the drivers slow down reducing their speed [33]. An important factor of traffic congestion uphill is that most drivers do not accelerate enough and consequently, they do not compensate instantaneously for the increase in resistance force resulting from the increase in gradient, which limits the acceleration of their vehicles. Considering that each vehicle has different capabilities and each driver different response times, gives a good explanation of the periodic formulation of stopand-go waves when the traffic demand is sufficiently high.

Here, a simple control logic in cooperation with the infrastructure is proposed. More specifically, we assume a central controller over an area, which imposes a unified $D S$ parameter value $\left(D S_{\text {coop }}\right)$ for all the vehicles within range. $D S_{\text {coop }}$ parameter regulates how much of the vehicle potential the driver will use (see Fig. 1), i.e. how hard the driver pushes the gas pedal. Since the vehicle models have different power specifications, it can be derived that each vehicle will have a different desired acceleration, proportional to the vehicle's individual capabilities. However, by asking each automated vehicles to use the same $D S_{\text {coop }}$ values, the controller unifies the driving profiles without setting explicit desired acceleration values per vehicle. This is a realistic strategy since some vehicle might not be physically able to comply 
with a suggested desired acceleration value due to power limitations.

Two cooperation strategies have been implemented here, which corresponds to two $D S_{\text {coop }}$ values, 0.8 and 0.6. The first value corresponds to more aggressive driving and thus higher acceleration values, while the second corresponds to a more conservative strategy for the vehicles entering the central controller's area.

\section{Methodology}

We propose 3 simulation scenarios, for each vehicle type. Each simulation experiment lasts $60 \mathrm{~min}$ with initial conditions according to capacity inflow. For each scenario, we run 100 simulation iterations per vehicle type with different random seeds in order to validate the results for different driving profile sequences in the simulations.

Initially, we create a pool of potential driving profiles as described in the Section 2.3. CVs are linked with 125 possible combinations, while AVs and Coop-AVs with 5 possible combinations (see Fig. 3.) Each time that a new vehicle enters the network, a driving profile is assigned to it from the corresponding pool of combinations. The distribution of the driving profiles entering the network is a normal distribution giving more probability to the middle (normal parameter values) and less probability to the extreme ones (timid or aggressive). The same distribution holds for all parameters of the driving profile $D P_{i}=\left\{C_{k}, G S_{t h, l}, D S_{m}\right\}$, the car, the gear shifting strategy and the driving style.

Three scenarios have been implemented in this work. Each scenario a pool of driving profiles is created based on different $\left\{C_{k}, G S_{t h, l}, D S_{m}\right\}$ value sets. The proposed cooperation logic is assessed with two possible cooperation values, $D S_{\text {coop }}$. Finally, CVs are simulated with reaction time equal to $1 \mathrm{~s}$, while the AVs and the CoopAVs with two different response times, one equal to the CVs reaction time and another one equal to the $90 \%$ of the average human reaction time.

\subsection{Scenario 1 - basic}

In the basic scenario, the $\left(D S, G S_{t h}\right)$ parameters take values between 0.1 (timid driver) and 0.9 (aggressive driver) for all the vehicle types CVs, AVs and Coop$\mathrm{AVs}$. For the last type, the cooperation value $D S_{\text {coop }}$, is set to 0.8 . This indicates that within the central controller's regulation area, all Coop-AVs will have free-flow $D S$ value, as indicated in the MFC model, equal to 0.8 . As described above, the driving profile assigned to the manually-driven vehicles uses a pool of 125 possible combinations with normal distribution around the average driving profiles. The corresponding number for AVs and Coop-AVs is 5 and also here, the distribution is a normal one around the average driving profile. As it can be inferred, this scenario implies that the driving behavior operational space (most aggressive and most timid drivers) is the same for all vehicle types ranging between 0.1 and 0.9 parameter values. However, AVs and CoopAVs induce homogeneity in the network because they can choose from only 5 possible $\left\{C_{k}, G S_{t h, l}, D S_{m}\right\}$ combinations. The goal here is to assess how reduction in the available driving behaviors impact the traffic flow in the network.

\subsection{Scenario 2 - homogenized AV driving profiles}

In the second scenario, the $\left(D S, G S_{t h}\right)$ parameters take values between 0.1 (timid driver) and 0.9 (aggressive driver) for the CVs, while for AVs and Coop-AVs, the corresponding range is 0.4 and 0.6. For Coop-AVs, the cooperation value $D S_{\text {coop }}$, is set to 0.8 , indicating the same driving behavior within the central controller's area as in the basic scenario. The lower $\left(D S, G S_{t h}\right)$ parameter values for automated vehicles indicate that extreme human driving behaviors (very timid or very aggressive) are filtered out. Consequently, it is assumed that automated controllers will have more homogenous free-flow acceleration values. The vehicles used in all simulations are the same and therefore, this scenario aims to study how the reduced driving profile variability and exclusion of outlier driving behaviors impact the status of the network.

\subsection{Scenario 3 - mild cooperation}

In the third scenario, similar to the previous one, the $\left(D S, G S_{t h}\right)$ parameters take values between 0.1 (timid driver) and 0.9 (aggressive driver) for the CVs, while for $\mathrm{AVs}$ and Coop-AVs, the corresponding range is 0.4 and 0.9. For Coop-AVs, the cooperation value $D S_{\text {coop }}$, is set to 0.6 , indicating a more relaxed cooperation within the central controller's area than in the previous scenarios. Due to the lower $D S_{\text {coop }}$ value, it can be expected that all automated vehicles will have lower accelerations than in previous scenarios. The goal here is to see if the expected cooperation benefits still hold for acceleration values that are lower, close to the average human driver.

The parameters of all scenarios are summarized in Table 1.

\section{Results}

This section presents the results and initiates a discussion on the three main dimensions of this work, the impact of increased homogeneity in the driving behavior domain due to automation, the potential benefits of cooperation and the role for anticipated reduced reaction time in future vehicles. The results of each scenario refer to 500 simulations with different parameter sets. Each simulation corresponds to $1 \mathrm{~h}$ and each simulation step is $1 \mathrm{~s}$. 
Table 1 Parameters per scenario and vehicle type

\begin{tabular}{llll}
\hline & Manual & AVs & Coop-AVs \\
\hline Scenario 1 & $\left(D S, G S_{t h}\right) \in[0.1,0.9]$ & $\left(D S, G S_{t h}\right) \in[0.1,0.9]$ & $\left(D S, G S_{t h}\right) \in[0.1,0.9]$ \\
& & & $D S_{\text {coop }}=0.8$ \\
Scenario 2 & $\left(D S, G S_{t h}\right) \in[0.1,0.9]$ & $\left(D S, G S_{t h}\right) \in[0.4,0.6]$ & $\left(D S, G S_{t h}\right) \in[0.4,0.6]$ \\
& & & $D S_{\text {coop }}=0.8$ \\
Scenario 3 & $\left(D S, G S_{t h}\right) \in[0.1,0.9]$ & $\left(D S, G S_{t h}\right) \in[0.4,0.6]$ & $\left(D S, G S_{t h}\right) \in[0.4,0.6]$ \\
& & $D S_{\text {coop }}=0.6$ \\
\hline
\end{tabular}

In the figures below, for each scenario and each vehicle type, 100 simulations were run with random seeds. Each seed practically changes the order of vehicles and drivers as they enter the network. As expected the performance of each vehicle type depends to a degree to the sequence of the vehicles/drivers that populate the network. For the three scenarios and the five vehicle types, the results are based to 1500 simulation. The duration of each test is $60 \mathrm{~min}$.

Figure $4 \mathrm{a}$ a) shows the speed over flow results for the Scenario 1, as well as the flow distributions for 100 simulations with random seeds per vehicle type (five distributions). In the speed over flow scatter plot, each dot corresponds to the average speed and average flow of all vehicles of the corresponding test run (random seed and vehicle type). Fig .4 b) illustrates the distributions of the average flow values per test run and vehicle type. Manually-driven and automated vehicles (with normal and reduced reaction time) have similar performances. The first observation is that the variation in the average flow for both vehicle types is high and obviously related to the sequence of the vehicles entering the network. More specifically, in a one-lane network, on carfollowing conditions the power dynamics of the leader affect all its followers. Each dot in Fig. 4 a) represents a simulation iteration with a random seed that defines that assignment of vehicles and driving profiles to the agents entering the network. For comparison purposes, the same set of seeds was used for all the simulations within all scenarios. In general AVs with response time equal to the reaction time of CVs seem to achieve lower speeds and lower flow values but the differences are not considered significant. AVs with reduced response time, as expected directly impact the capacity of the network and thus perform slightly better than AVs with normal response time. The most interesting results refer to the performance of Coop-AVs. The proposed simple cooperation logic seems to increase significantly both the average speed and the average flow in the network for any random seed. Moreover, the variation in the average speed and flow values among different simulation iterations decreases dramatically, reducing the uncertainty regarding the simulation results. Coop-AVs with reduced response time perform on average slightly worse than Coop-AVs with normal response time. For specific seeds, they achieve the highest average flow values but there is large variation among different tests. In the proposed car-following model the reduction in the response time is simulated by increasing the wave speed and thus the propagation of any perturbation downstream. This can be an explanation for these results. In the first scenario, there is high uncertainty on the expected results as it is shown in Fig. 4 b). Only Coop-AVs have a very narrow distribution implying consistency on their performance.

Figure 5 illustrates the corresponding results on the second scenario. In this scenario, the pool of available vehicles and drivers for AVs and Coop-AVs is constrained and therefore the distribution of vehicles and drivers is more homogenous. Since, the same set of random seeds was used, the results for CVs are the same as in Fig. 4. In all simulation iterations within the second scenario, AVs perform much better than Scenario 1, reaching much greater average speed and flow values. In fact, the performance of AVs approaches the one from

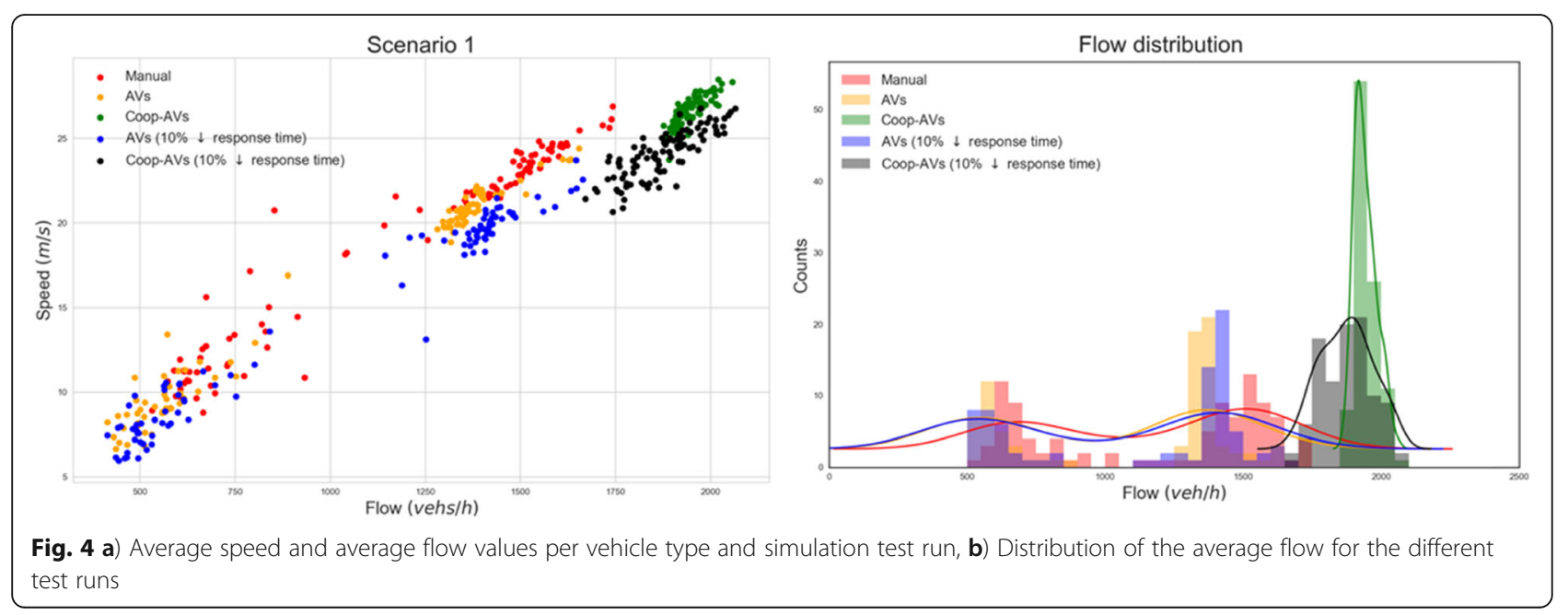




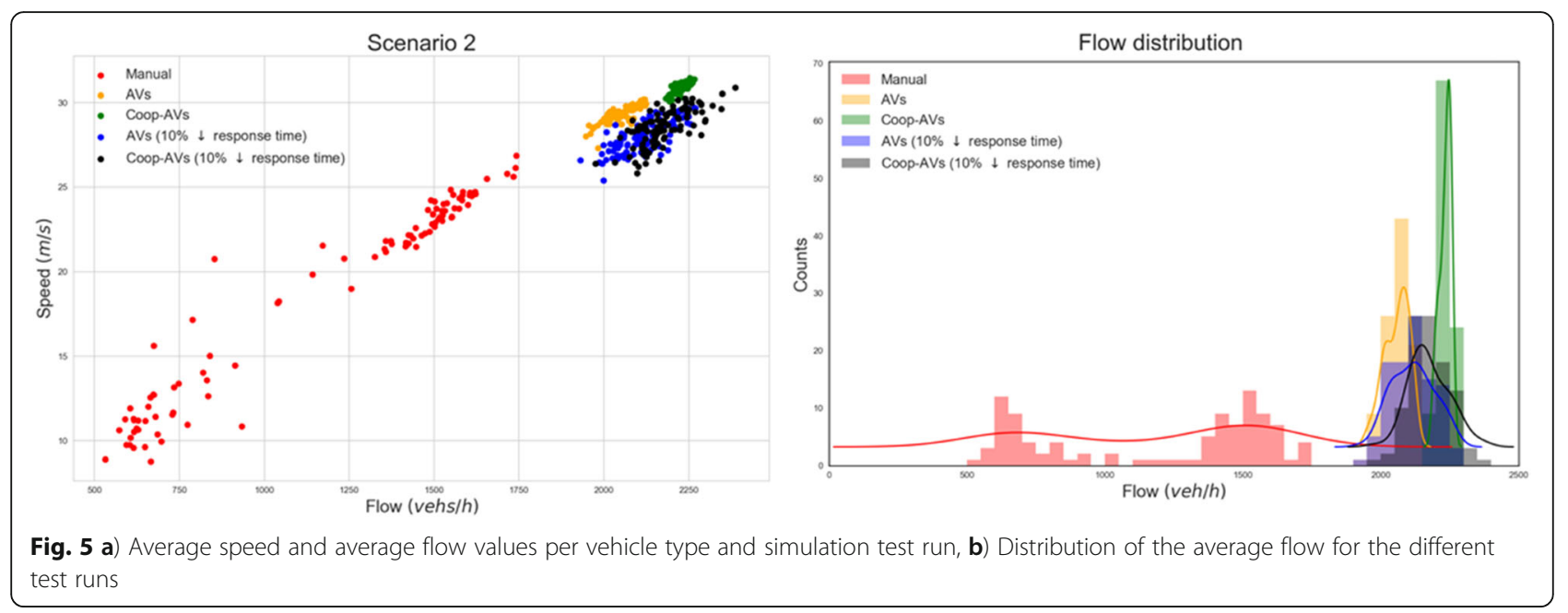

Coop-AVs, which is the best among the three type of vehicles. This is the result of reducing the variability in the driving profiles, and limiting the values of $\left(D S, G S_{t h}\right)$ parameters in the range between 0.4 and 0.6. These values practically mean that the driving behaviors of AVs and Coop-AVs is more homogenous, i.e. not too aggressive and not too conservative. The DS parameter value is directly related with the power capabilities of the vehicles, and thus the autonomous drivers at any given speed exploit between $40 \%$ and $60 \%$ of the vehicle's acceleration potential. The $G S_{t h}$ parameter value is linked with the rpm values of the engine when the driver shifts gears (for more details please see [18]. From the results, it can be inferred that an important factor for the impact of AVs, will be their homogenous operation on the road or not. Homogeneity in the controllers' acceleration can dramatically improve the status of such a network. This is clear from both Fig. 5 a) and b). In Fig. 5 b) the distributions of all types but human-driven vehicles are quite narrow implying consistency for all vehicle orders in the network. Reduced response time for both AVs and Coop-AVs for some test leads to higher average flow values. However, as in Scenario 1, the variability among different simulation tests increases and thus there is less consistency in the potential benefit.

The last scenario focuses on the impact of the cooperation control logic of the Coop-AVs. Coop-AVs have the best performance in Scenarios 1 and 2. However, the cooperation can be either aggressive or more conservative depending on the values of the parameter $D S_{\text {coop }}$. In the first two scenarios, this parameter is equal to 0.8 , meaning the within the cooperation region, Coop-AVs exploit a lot of their power capabilities and thus produce high acceleration values. In that sense, it is normal that the situation is vastly improved in comparison with $\mathrm{AVs}$ and certainly CVs. In this scenario, we lower the cooperation value to 0.6 , in order to observe if the benefits of the
V2I cooperation still hold and up to which extend. Lowering $D S_{\text {coop }}$ value means that some drivers will be slower compared to the second scenario. However, by adopting a global acceleration strategy we achieve the homogenization of the accelerations within the range of the central controller. The results are shown in Fig. 6 . Even with lowered cooperation parameter, Coop-AVs still have the best performance. On average, the results are similar to the second scenario.

Finally, regarding the impact of homogenization in traffic flow, on the capacity of the network, the results discussed above show clearly that more homogenous driving behaviors lead to more homogenous flows and greater capacity. Cooperation with the infrastructure certainly helps, while the anticipated reduction in response times of the vehicle does not seem to play a crucial role. Table 2 shows the average speed and flow values for the 100 simulation tests per scenario and per vehicle type presented above. This table provides an easy way for assessment and comparison between different runs.

Figure 7 illustrates the vehicle's average speed for three random simulation tests, for Scenarios 1, 2 and 3 and the corresponding empirical observations from the NGSIM database [24]. The $x$-axis depicts the order of the vehicles as they enter the network, while the $y$-axis is their average speed after they exit the network. The goal is to show how a perturbation propagates the information upstream and creates oscillations on the basis of the vehicles' average speeds. In the first three figures, different vehicle types introduce oscillations created from the uphill. CVs are the most unstable with the larger oscillation amplitudes. For the second and third scenarios, that the vehicles and drivers for the automated vehicles are more homogenous, the oscillation amplitudes are lower and the period is larger. Coop-AVs in all scenarios have the best performance. Decrease in the response time 

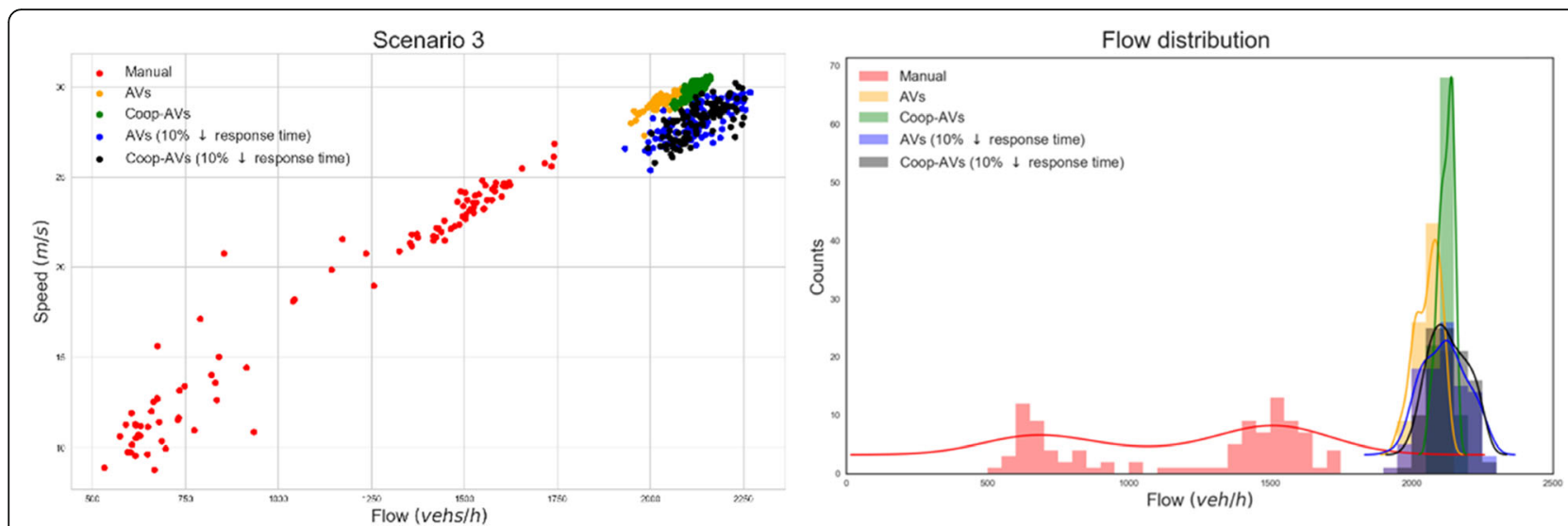

Fig. 6 a) Average speed and average flow values per vehicle type and simulation test run, b) Distribution of the average flow for the different test runs

propagates the perturbation information faster upstream facilitating the formation of average speed oscillations. Finally, Fig. 7 d) shows the corresponding graph for a part of the NGSIM database for the second, third and fourth lanes, only to demonstrate how such oscillations are visible in empirical measurements. It is interesting to observe visually similar patterns as in the simulation results. Of course, further study is necessary in addition to the preliminary results presented here, however this is considered outside the scope of the present paper.

\section{Conclusions}

Vehicle automation and cooperation is expected to bring new driving patterns in future networks and more homogeneity regarding individual driving behaviors. This work presents a microsimulation study on the topic on an uphill network. Uphill road sections are responsible for capacity bottlenecks in freeway networks. Variability in the driving behaviors seems to play a key role on the severity of the bottlenecks and the consequent stop-and-go waves. It has been reported in the literature that homogenized flows can mitigate or even solve such problems. The novelty of the proposed approach is that

Table 2 Average results for speed and flow per vehicle type, scenario and response speed. Abbreviations N.RT and R.RT mean Normal Response Time and Reduced Response Time respectively

\begin{tabular}{|c|c|c|c|c|c|c|c|}
\hline & & \multicolumn{2}{|c|}{ Scenario 1} & \multicolumn{2}{|c|}{ Scenario 2} & \multicolumn{2}{|c|}{ Scenario 3} \\
\hline & & $\overline{N . R T}$ & R.RT & N.RT & R.RT & N.RT & R.RT \\
\hline \multirow[t]{2}{*}{ CVs } & Speed & 18.83 & - & 18.83 & - & 18.83 & - \\
\hline & Flow & 1183 & - & 1183 & - & 1183 & - \\
\hline \multirow[t]{2}{*}{ AVs } & Speed & 15.84 & 14.72 & 29.40 & 28.00 & 29.40 & 28.00 \\
\hline & Flow & 1013 & 1026 & 2057 & 2117 & 2057 & 2117 \\
\hline \multirow[t]{2}{*}{ Coop-AVs } & Speed & 26.56 & 24.08 & 30.99 & 28.54 & 29.93 & 28.15 \\
\hline & Flow & 1942 & 1875 & 2234 & 2175 & 2121 & 2130 \\
\hline
\end{tabular}

demonstrates the impact of anticipating homogeneity in vehicle and driver behaviors using a common hybrid car-following model that takes explicitly into account the variability in the vehicle dynamics of different vehicles and the driving behavior of different drivers. An interesting addition to existing microsimulation studies is that the different vehicle technologies are simulated using the same car-following model. Only the free-flow part of this model is parametrized to reflect different homogeneity levels depending on the scenario, while the rest model parameters remain independent of the vehicle type.

The proposed methodology applies three simulation scenarios, for each vehicle type, Conventional vehicles (CVs), AVs and Coop-AVs. Each simulation experiment lasts $60 \mathrm{~min}$ with initial conditions according to capacity inflow. For each scenario, we run 100 simulation iterations per vehicle type with different random seeds in order to see the impact for different vehicle and driving profile sequences in the simulations. Results confirm that homogeneity in terms of vehicle dynamics and driver behavior can play an important role towards improving traffic flow. Furthermore, the cooperation with the infrastructure can limit high variations in the vehicles' accelerations and thus potential traffic jams.

The main contributions and results of the proposed work can be summarized as follows:

- Simulation of CVs, AVs and Coop-AVs in uphill freeway segments and analysis of driving homogeneity impact using a car-following model that reproduces realistic vehicle dynamics and driver profiling. The study focuses on three main dimensions, the driving behavior, the reaction and response time, the vehicle cooperation with the infrastructure.

- Homogeneity in all possible driving patterns within a network can significantly facilitate traffic flow. 


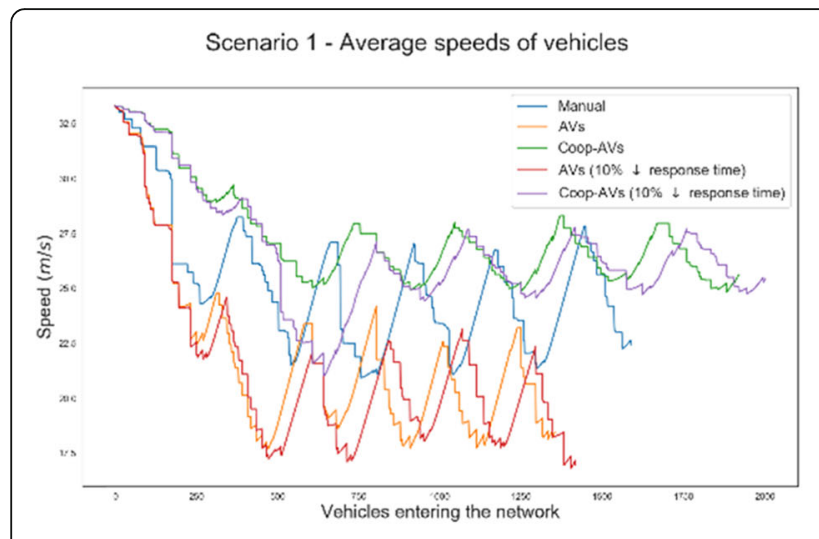

(a)

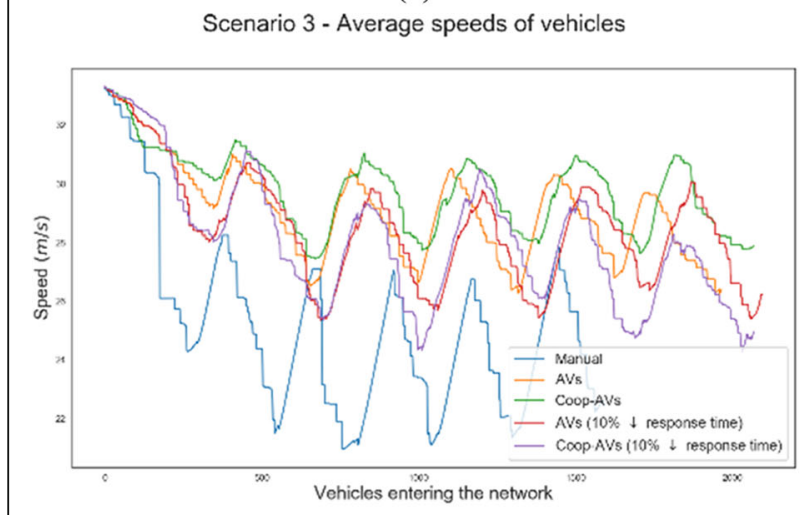

(c)
Scenario 2 - Average speeds of vehicles

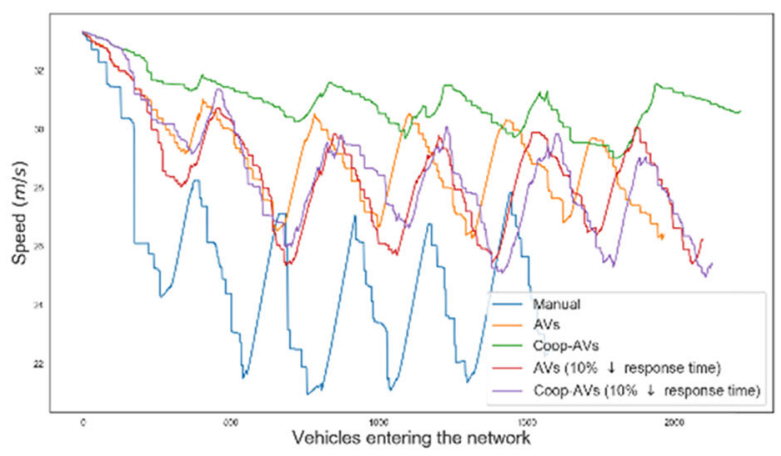

(b)

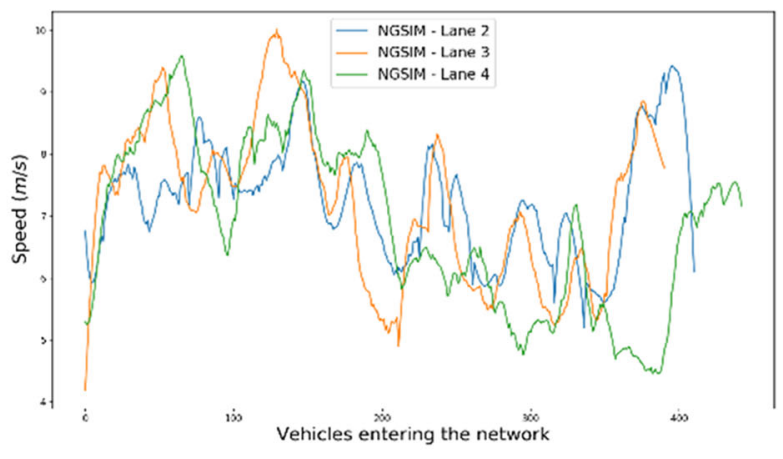

(d)

Fig. 7 Average speed per vehicle, for all the vehicles entering the network. Sample simulation tests for Scenarios 1, 2 and 3 are shown in figures a), b) and c). Figure d) shows empirical observations in three lanes in a congested segment of the NGSIM database

Homogeneity can refer to either vehicles dynamics, meaning automated controllers with similar responses, or driving style, meaning aggressive or timid driving patterns are excluded.

- Cooperation with the infrastructure can benefit the status of the network. After homogeneity, this is the second most important factor. It can play an essential role in future networks if driving heterogeneity on the roads persist.

- Lower response times increase the theoretical capacity of the network but also the propagation of perturbation information upstream. Thus, for large perturbations the network becomes saturated faster. The anticipated reduced response times of AVs are not expected to have a significant impact on traffic flow.

In future work, the present work will be conducted with the extended version of the MFC model for electric powertrains [12], since electrified vehicle have different dynamics. A publicly available library of the MFC model can be found online (https://pypi.org/project/co2mpasdriver/). Additionally, it would be interesting to validate the above-mentioned observations in a more realistic network cross-validating the results here using additional car-following models, where their free-flow part will be substituted by the proposed MFC model. Furthermore, it would be interesting to understand how different gradients or type of perturbations affect the magnitude of the impact that homogeneity has on traffic flow. Finally, further and more systematic assessment of the capability of such models to reproduce empirical observation is necessary.

\section{Acknowledgements}

This work was based on the collaboration agreement between the Joint Research Centre of the European Commission and the LICIT laboratory, Gustave Eiffel University .

\section{Authors' contributions}

$M M$ and $L L$ coordinated the study and designed the methodology. $\mathrm{MM}$ and $\mathrm{KM}$ performed the data analysis and simulation. The draft manuscript preparation was made by MM. LL, KM and $\mathrm{BC}$ contributed to the writing of the manuscript. All authors read and approved the final manuscript.

Funding

Not applicable. 


\section{Availability of data and materials}

Not applicable.

\section{Competing interests}

The authors declare that they have no competing interests.

\section{Author details}

${ }^{1}$ European Commission, Joint Research Centre, Ispra, VA, Italy. ${ }^{2}$ Laboratoire Ingénierie Circulation Transport LICIT (INRETS/ENTPE), Gustave Eiffel University, Lyon, France.

Received: 22 July 2019 Accepted: 25 February 2020

Published online: 17 March 2020

\section{References}

1. Alonso Raposo, M., Ciuffo, B., Makridis, M., Thiel, C., 2017. The r-evolution of driving: From connected vehicles to coordinated automated road transport (C-ART). Publications Office of the European Union. https://doi.org/10.2760/ 666294 (ePub).

2. Ciuffo, B., Makridis, M., Toledo, T., \& Fontaras, G. (2018). Capability of current Car-following models to reproduce vehicle free-flow acceleration dynamics. IEEE Trans Intell Transp Syst, 1-10 https://doi.org/10.1109/TITS.2018.2866271.

3. Daganzo, C. F. (2006). In traffic flow, cellular automata=kinematic waves. Transp. Res. Part B Methodol., 40, 396-403 https:/doi.org/10.1016/j.trb.2005.05.004.

4. Daganzo, C. F. (2005). A variational formulation of kinematic waves: Solution methods. Transp. Res. Part B Methodol., 39, 934-950 https://doi.org/10.1016/j. trb.2004.05.003.

5. European Commission, 2019. The future of road transport [WWW document]. EU Sci. Hub - Eur. Comm. URL https://ec.europa.eu/jrc/en/ publication/eur-scientific-and-technical-research-reports/future-roadtransport (accessed 8.23.19).

6. EUROPEAN COMMISSION, 2017. EUROPE ON THE MOVE - an agenda for a socially fair transition towards clean, competitive and connected mobility for all.

7. Fadhloun, K., Rakha, H., Abdelkefi, A., Loulizi, A., 2017. An enhanced RakhaPasumarthy-Adjerid Car-following model accounting for driver behavior. Presented at the Transportation Research Board 96th annual MeetingTransportation research board.

8. Goñi-Ros, B., Knoop, V. L., Takahashi, T., Sakata, I., van Arem, B., \& Hoogendoorn, S. P. (2016). Optimization of traffic flow at freeway sags by controlling the acceleration of vehicles equipped with in-car systems. Transp. Res. Part C Emerg. Technol., 71, 1-18 https://doi.org/10.1016/j.trc.2016.06.022.

9. Goñi-Ros, B., Schakel, W. J., Papacharalampous, A. E., Wang, M., Knoop, V. L., Sakata, I., van Arem, B., \& Hoogendoorn, S. P. (2019). Using advanced adaptive cruise control systems to reduce congestion at sags: An evaluation based on microscopic traffic simulation. Transp. Res. Part C Emerg. Technol., 102, 411-426 https://doi.org/10.1016/j.trc.2019.02.021.

10. Green, M. (2000). How long does it take to stop? Methodological Analysis of Driver Perception-Brake Times Transp Hum Factors, 2, 195-216 https:/doi.org/ 10.1207/STHF0203_1.

11. He, Y., Ciuffo, B., Zhou, Q., Makridis, M., Mattas, K., Li, J., Li, Z., Yan, F., Xu, H., 2019 Adaptive cruise control strategies implemented on experimental vehicles: A review. IFAC-pap., 9th IFAC symposium on advances in automotive control AAC 2019 52, 21-27. https://doi.org/10.1016/j.ifacol.2019.09.004.

12. He, Y., Makridis, M., Mattas, K., Fontaras, G., Ciuffo, B., Xu, H.: "Enhanced MFC: Introducing Dynamics of Electrified Vehicles for Free Flow Microsimulation Modeling" to appear, Transportation Research Record, March 2020.

13. Huang, Y.-X., Jiang, R., Zhang, H., Hu, M.-B., Tian, J.-F., Jia, B., \& Gao, Z.-Y. (2018). Experimental study and modeling of car-following behavior under high speed situation. Transp. Res. Part C Emerg. Technol., 97, 194-215 https://doi.org/10.1016/j.trc.2018.10.022.

14. Kesting, A., Treiber, M., Schönhof, M., \& Helbing, D. (2008). Adaptive cruise control design for active congestion avoidance. Transp. Res. Part C Emerg. Technol., 16, 668-683 https://doi.org/10.1016/j.trc.2007.12.004.

15. Laval, J. A., Toth, C. S., \& Zhou, Y. (2014). A parsimonious model for the formation of oscillations in car-following models. Transp Res Part $B$ Methodol, 70, 228-238 https://doi.org/10.1016/j.trb.2014.09.004.

16. Leclercq, L., Laval, J.A., Chevallier, E., 2007. The Lagrangian coordinates and what it means for first order traffic flow models. Presented at the transportation and traffic theory 2007. Papers selected for presentation at ISTTT17Engineering and physical sciences research council (great Britain) Rees
Jeffreys road FundTransport research FoundationTMS ConsultancyOve Arup and partners, Hong KongTransportation planning (international) PTV AG.

17. Lighthill, M. J., \& Whitham, G. B. (1955). On kinematic waves. II A Theory of Traffic Flow on Long Crowded Roads Proc R Soc Lond Ser Math Phys Sci, 229, 317-345.

18. Makridis, M., Fontaras, G., Ciuffo, B., \& Mattas, K. (2019a). MFC free-flow model: Introducing vehicle dynamics in microsimulation. Transp Res Rec, 2673, 762-777 https://doi.org/10.1177/0361198119838515.

19. Makridis, M., Mattas, K., Borio, D., Giuliani, R., Ciuffo, B., 2018. Estimating reaction time in adaptive cruise control system, in: 2018 IEEE intelligent vehicles symposium (IV). Presented at the 2018 IEEE intelligent vehicles symposium (IV), pp. 1312-1317. https://doi.org/10.1109/IVS.2018.8500490.

20. Makridis, M., Mattas, K., Ciuffo, B., 2019b. Response time and time headway of an adaptive cruise control. An Empirical Characterization and Potential Impacts on Road Capacity IEEE Trans Intell Transp Syst 1-10. https://doi.org/ 10.1109/TITS.2019.2948646.

21. Makridis, M., Mattas, K., Ciuffo, B., Fontaras, G., 2020. The impact of automation and connectivity on traffic flow and $\mathrm{CO} 2$ emissions. A detailed microsimulation study.

22. Mattas, K., Makridis, M., Hallac, P., Raposo, M. A., Thiel, C., Toledo, T., \& Ciuffo, B. (2018). Simulating deployment of connectivity and automation on the Antwerp ring road. IET Intell Transp Syst, 12, 1036-1044 https://doi.org/10. 1049/iet-its.2018.5287.

23. Milanes, V., Shladover, S. E., Spring, J., Nowakowski, C., Kawazoe, H., \& Nakamura, M. (2014). Cooperative adaptive cruise control in real traffic situations. IEEE Trans Intell Transp Syst, 15, 296-305 https://doi.org/10.1109/ TITS.2013.2278494.

24. Montanino, M., \& Punzo, V. (2013). Making NGSIM data usable for studies on traffic flow theory: Multistep method for vehicle trajectory reconstruction. Transp Res Rec, 2390, 99-111 https://doi.org/10.3141/2390-11.

25. Ngoduy, D. (2013). Instability of cooperative adaptive cruise control traffic flow: A macroscopic approach. Commun Nonlinear Sci Numer Simul, 18, 2838-2851 https://doi.org/10.1016/j.cnsns.2013.02.007.

26. Ros, B.G., Knoop, V.L., Arem, B. van, Hoogendoorn, S.P., 2012. Reducing congestion at uphill freeway sections by means of a gradient compensation system, in: 2012 IEEE intelligent vehicles symposium. Presented at the 2012 IEEE intelligent vehicles symposium, pp. 191-198. https://doi.org/10.1109/ IVS.2012.6232150.

27. Soriguera, F., Martínez, I., Sala, M., \& Menéndez, M. (2017). Effects of low speed limits on freeway traffic flow. Transp Res Part C Emerg Technol, 77, 257-274 https://doi.org/10.1016/j.trc.2017.01.024.

28. Summala, H. (2000). Brake reaction times and driver behavior analysis. Transp Hum Factors, 2, 217-226 https://doi.org/10.1207/STHF0203_2.

29. Talebpour, A., \& Mahmassani, H. S. (2016). Influence of connected and autonomous vehicles on traffic flow stability and throughput. Transp. Res. Part C Emerg. Technol., 71, 143-163 https://doi.org/10.1016/j.trc.2016.07.007.

30. Thiel, C., Schmidt, J., Van Zyl, A., \& Schmid, E. (2014). Cost and well-to-wheel implications of the vehicle fleet $\mathrm{CO} 2$ emission regulation in the European Union. Transp Res Part Policy Pract, 63, 25-42 https://doi.org/10.1016/j.tra. 2014.02.018.

31. Tsiakmakis, S., Fontaras, G., Ciuffo, B., \& Samaras, Z. (2017). A simulation-based methodology for quantifying European passenger car fleet CO2 emissions. Appl Energy, 199, 447-465 https://doi.org/10.1016/j.apenergy.2017.04.045.

32. Wu, C., Yu, D., Doherty, A., Zhang, T., Kust, L., \& Luo, G. (2017). An investigation of perceived vehicle speed from a driver's perspective. PLOS ONE, 12 https://doi.org/10.1371/journal.pone.0185347.

33. Yishui, S., Wei, C., \& Fang, L. (2015). Research on bottleneck effect of tunnel in steep uphill of city highway, in: 2015 international conference on transportation information and safety (ICTIS). In Presented at the 2015 international conference on transportation information and safety (ICTIS) (pp. 133-137) https://doi.org/10.1109/ICTIS.2015.7232065.

\section{Publisher's Note}

Springer Nature remains neutral with regard to jurisdictional claims in published maps and institutional affiliations. 